\title{
La intervención arquitectónica de 2018 en la Torre de la Sal de Cabanes (Castellón)
}

The architectural intervention of 2018 in the Salt Tower of Cabanes (Castellón)

\section{Jaime Prior y Llombart}

Departamento de Proyectos Arquitectónicos - Escuela Técnica Superior de Arquitectura - Universitat Politècnica de València, Valencia, Spain, prior@ctac.es

\begin{abstract}
Salt Tower is a complete defensive structure located in the town of Cabanes, in Castellón, at the north of the Spanish Valencian Community: "Torre de la Sal", a link in the long coastal defense chain. The architectural intervention has basically consisted of the external consolidation of the masonry walls, especially of two of them; the protection of water infiltration and the replacement of the extemporaneous structure of the existing balcony. Internally, all the coatings have been renewed, eliminating aggressive applications and providing it with an electrical installation.

The works have been adjusted to the strictest criteria from a quality point of view and have been a consequence of the findings obtained in the research phase that have determined the final result, varying in some cases those provided for in the intervention project.

The results received after the intervention are presented, waiting to proceed with the intensive excavation of the immediate surroundings of the tower, located on a powerful buried urban structure, lack of excavation and submerged in the sea.
\end{abstract}

Keywords: Salt, tower, defending, coast, restoration, intervention, architecture.

\section{Introducción}

La Torre de la Sal en Cabanes, Castellón, es un elemento considerado Bien de Interés Cultural según la vigente legislación de Patrimonio de la Comunidad Valenciana. Se sitúa en las coordenadas Latitud $40^{\circ} 77^{\prime} 51.18^{\prime \prime} \mathrm{N}$ Longitud $0^{\circ}$ 9'47.09'E. Aparece referenciado en el Inventario General del Patrimonio Cultural Valenciano con el código 12.05.033-007 y con referencia de anotación en el Ministerio R-I-51-0010751 de fecha $24 / 04 / 02$.

Está situada en el extremo sur del Parque Natural del Prat de Cabanes, sobre un yacimiento ibérico de gran interés.
De forma paralelepipédica y másica con planta cuadrada tiene las diagonales prácticamente orientadas en las cuatro direcciones cardinales. Tiene una superficie útil interior cerrada de $47,92 \mathrm{~m}^{2}$ y por el contrario dispone de una superficie construida total de $135,54 \mathrm{~m}^{2}$.

Es una construcción de mampostería ordinaria, de dimensiones $6,60 \times 6,60 \mathrm{~m}$, tiene las aristas y algunos huecos resueltos con sillería concertada. Con muros de un considerable espesor (160/135/115 cm según las plantas), resuelve sus dos forjados principales mediante bóvedas de cañón con directriz alterna en una planta respecto de la otra. 
Las características de la torre y el proyecto de intervención quedan totalmente determinados y descritos en la comunicación realizada en el Congreso FORTMED 2018 en Turín (Prior. 2018, pp. 823-830). El objeto de la actual comunicación es dar cuenta del resultado de la intervención realizada en base a aquél proyecto y a los cambios adoptados por las características de los hallazgos.

\section{La intervención exterior}

Exteriormente la intervención ha consistido en una tarea de limpieza intensiva por chorro de agua de los revestimientos en las caras noroeste y suroeste donde los existentes se encontraban en un estado bastante aceptable.

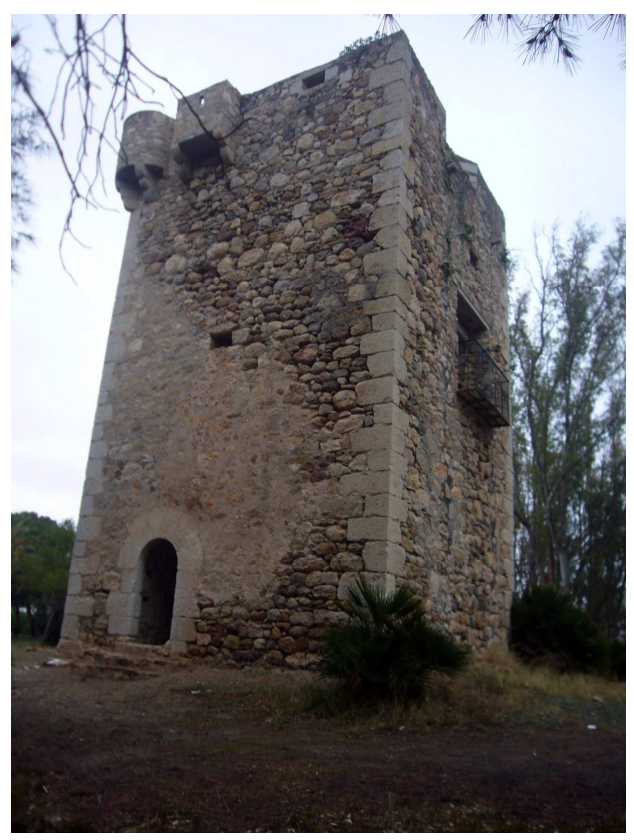

Fig. 1. Ángulo Sur antes de la intervención. Se observa la estructura del balcón suprimido por extemporáneo, añadido en la década de 1960 (Autor, 2016).

Los paramentos eran homogéneos y se encontraban en condiciones buenas de planicidad. Tan solo se ha retacado puntualmente algunos fallos mínimos y se ha limpiado de manera mecánica los paramentos de la cantonera de piedra del ángulo norte, muy dañada por una inclusión de moho.
En el frente sureste, el que contiene la puerta de entrada, se ha procedido a eliminar completamente el enfoscado parcial de mortero de cemento que cubría una gran parte de su superficie. Se ha eliminado el mortero, cepillado enérgicamente la piedra y posteriormente se ha revestido con un mortero de cal, dispuesto a cara enrasada, al igual que están los otros dos paramentos antes comentados.

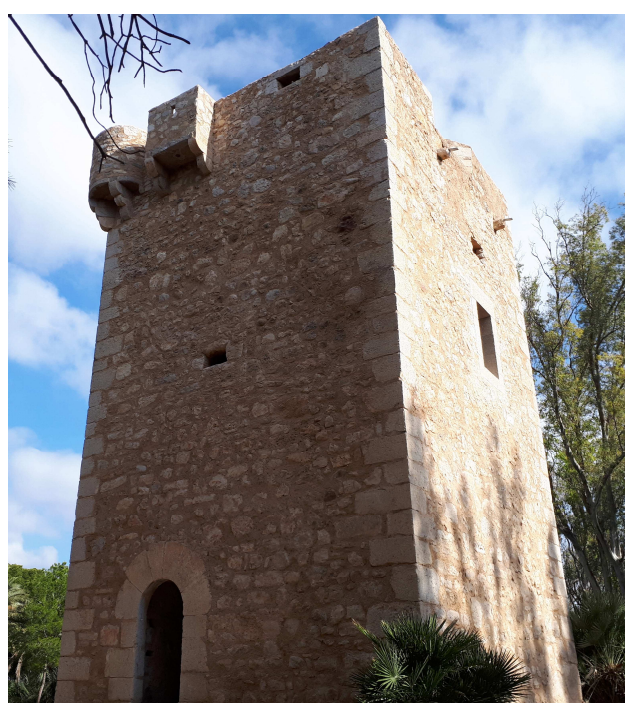

Fig. 2. Ángulo sur después de la intervención de 2018 (Autor, 2018).

Se ha restaurado la puerta principal de entrada, recomponiendo la cerradura y limpiando los herrajes.

Como acabado superficial de la madera, después de tratarla, se ha colocado una plancha de acero, dotándola así de unas condiciones extraordinarias frente a su durabilidad y a la acción de los vandalismos que pudieran sobrevenirle. Por supuesto se han mantenido los herrajes existentes.

En la parte exterior se ha habilitado el espacio colateral al acceso, recuperando los niveles de ocupación iniciales y descubriendo los sillares del umbral, que se mantenían ocultos por la tierra. Sobre uno de esos mampuestos se ha depositado la señalización de la torre mediante una placa de acero inoxidable grabada con corte de agua. 

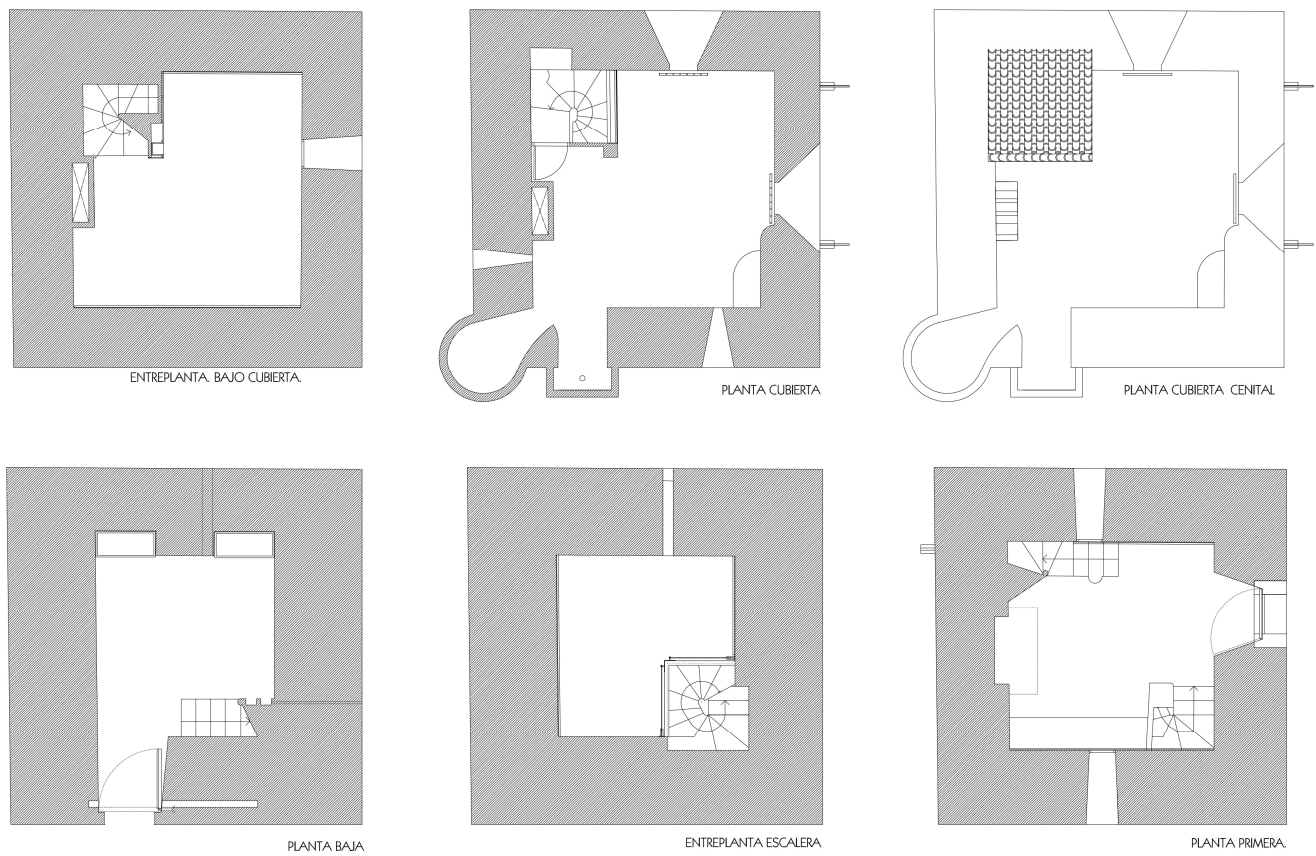

Fig. 3. Plantas de la Torre después de la intervención. El impacto planimétrico de las intervenciones han sido mínimas centradas únicamente en la recomposición del hueco donde se situaba el balcón abierto en el frente que se enfrentaba al mar (Proyecto F. O., J. Prior \& V.M. Llombart).

La mayor intervención sobre los cuatro paramentos de la torre se centra en el frente nordeste donde existía un grotesco balcón y un hueco totalmente fuera de escala y de configuración.

Los materiales que se habían utilizado para construirlo y la carpintería que cerraba dicho hueco, permiten datar esta obra revertida a principios de los años sesenta del pasado siglo.

La cerrajería del balcón no tenía ningún valor especial pero dos elementos atornillados a él pensados para soporte de teas, probablemente reaprovechados también, se han mantenido con un nuevo soporte, fijándolos a las paredes de la planta primera.

Este era, al mismo tiempo, el frente más dañado. Poder contemplarlo directamente desde el andamio, permitió identificar gran cantidad de mampuestos que se encontraban totalmente sueltos, sin argamasa y amenazando un peligro de desprendimiento inminente.

Se han eliminado todas las inclusiones vegetales y las biológicas (nidos de ave y reptiles) antes de proceder a la eliminación del balcón y a la recomposición, según el proyecto, de un hueco resuelto con sillería concertada.

El balcón no tenía ningún interés, pero sí las piezas cerámicas que resolvían el intradós de la retícula metálica de su parte volada. Estas piezas, de factura manual, eran reaprovechadas de otro lugar indeterminado y se han recuperado, limpiado y colocado en un soporte especialmente diseñado para ello en una de las paredes del interior de la planta primera.

El resto de elementos metálicos del balcón se han reaprovechado para resolver la seguridad de las troneras de artillería en cubierta.

De la misma manera que los otros frentes, después de realizadas las operaciones de recomposición de la mampostería, el frente se ha rejuntado y revestido con mortero de cal. Se ha dado una capa superficial de entonación superficial para igualar la cromaticidad de los diferentes paramentos. 


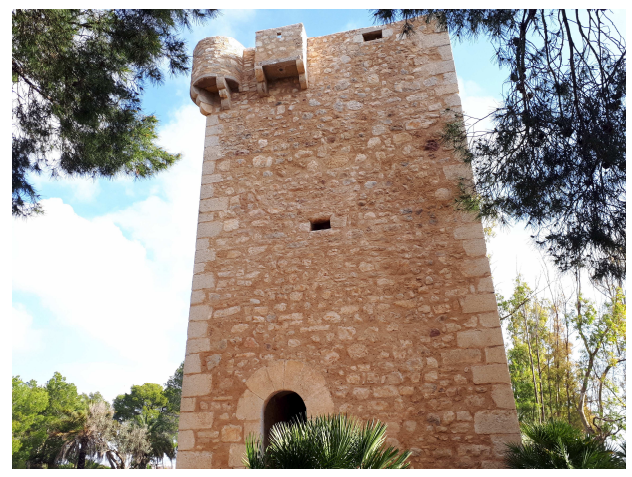

Fig. 4. Lienzo suroeste después de la intervención (Autor, 2018).

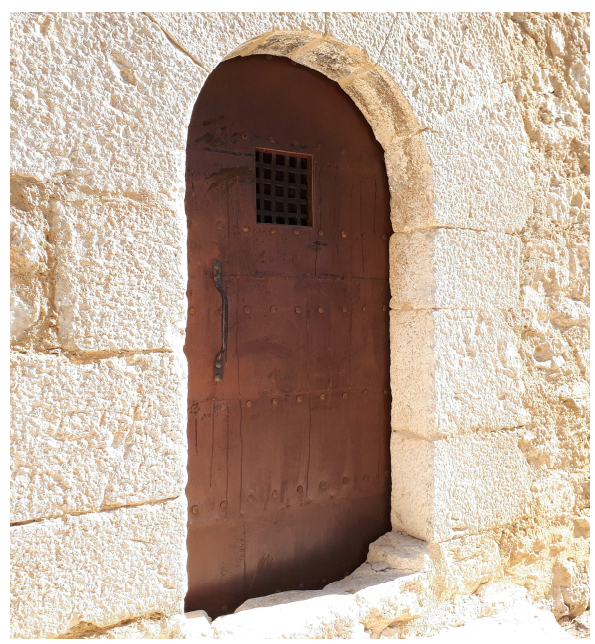

Fig. 5. Puerta acabada, protegida con lámina de acero (Autor, 2018).

\section{La cubierta}

En los paramentos de cubierta, después de eliminar las inclusiones vegetales, los elementos oxidados de zunchado y los añadidos de mortero de cemento se ha procedido a su homogeneización mediante el rejuntado con mortero de cal, diferenciando y manteniendo las diferentes facturas de las fábricas allí existentes.

La cubierta manifiesta como ningún punto las continuas manipulaciones y reformas que la torre ha sufrido con el paso del tiempo.

En los zunchados del garitón y el matacán situados al sur, se ha sustituido el oxidado acero del armado de coronación por redondos de fibra de vidrio antes de recomponer el acabado con mortero de cal. Se ha recuperado totalmente la volumetría preexistente.
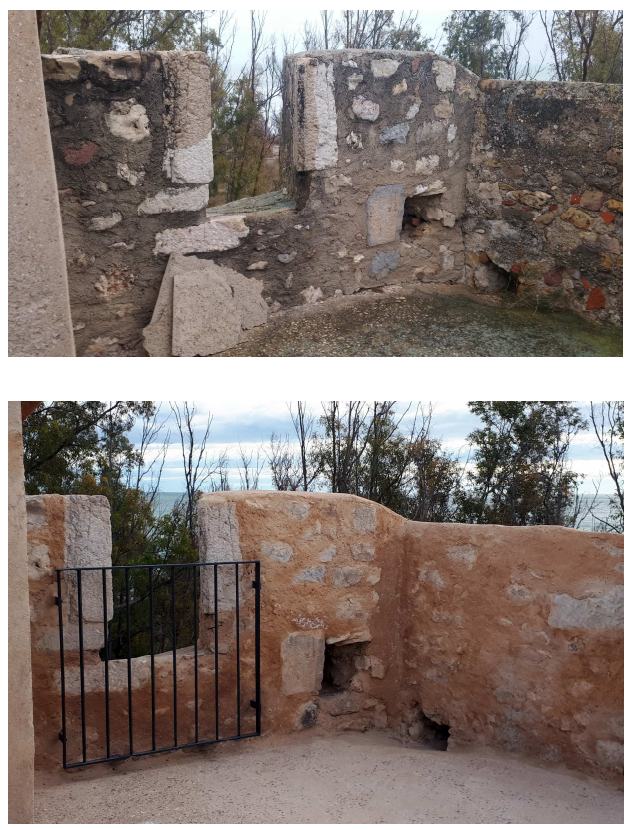

Fig. 6. Visión anterior / posterior de un ángulo de la cubierta (Autor, 2016 y 2018).

En el pavimento de la cubierta, al comprobar que existía una capa superficial de mortero de fecha muy reciente, se ha eliminado completamente ésta y se ha aplicado la capa de impermeabilización. A continuación se ha restituido la capa de protección de la impermeabilización con hormigón de cal de baja granulometría. Con ello se ha conseguido un acabado bastante unitario para el conjunto al entonar con el tratamiento de las paredes.

El sistema de evacuación de aguas se ha mantenido el existente, prolongando las actuales gárgolas de piedra con unos elementos de plancha de cinc para conseguir el vertido de las escorrentías en un punto más alejado de las fábricas; uno de los motivos de su alto deterioro debido a la erosión por lavado del aglomerante.

Por último, se ha sustituido completamente la puerta de la garita del acceso y se ha consolidado la forma que derivaba de la caída del tabique lateral en la caseta de escalera, disponiendo una 
carpintería de acero con vidrio opal que permite iluminar y ventilar continuamente este espacio alto de llegada.

\section{Planta baja}

Inicialmente la planta baja se encontraba resuelta con un pavimento de hormigón muy burdo, con mucha pendiente hacia la puerta de entrada.

Al eliminar esta capa superior apareció un pavimento previo compuesto por un enmorrellat de pieza gruesa con una inclinación bastante menor que la que tenía el hormigón precedente. La aparición de este pavimento demostraba a las claras el uso como caballerizas de este bajo, ya que no era un pavimento para tránsito humano.

La decisión de mantenerlo, consultada al Ayuntamiento de Cabanes y a la Dirección General de Patrimonio de la Generalitat Valenciana, comportaba anular la posibilidad de usar el espacio rehabilitado como oficina de turismo, como era intención.

Ello representó también reconsiderar las condiciones de accesibilidad del monumento, en beneficio de mantener su integridad.

En la obra, se procedió a eliminar la capa de hormigón, a limpiar el pavimento y rejuntarlo con mortero de cal en seco, humedeciendo después por aspersión para su fraguado y fijación.

Las paredes de la planta baja se sanearon completamente, eliminando la pintura y el recubrimiento dañado; procediendo a continuación a rejuntar las partes bajas y a repasar las bóvedas.

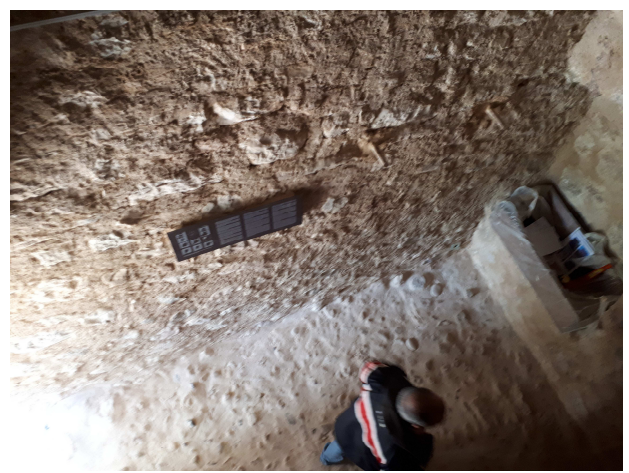

Fig. 7. Acabado de planta baja. Visión del pavimento encontrado (Autor, 2018).
En una de las paredes de la planta baja, lateralmente a la entrada se ha colocado una breve explicación del carácter de la torre y del alcance da la intervención arquitectónica planteada.

\section{Planta primera}

En la planta primera, la supresión de la bancada existente, que estaba sobrepuesta al pavimento de ladrillo, puso de manifiesto un nivel de ocupación anterior, materializado en un pavimento de mortero de cal alisado.

Este acabado debe ser el primer pavimento que existió en la torre, con una pendiente pronunciada hacia la escalera, que tendría una configuración diferente a la actual. El hallazgo se ha dejado visto, sin recubrir, protegiéndolo con una imprimación incolora consolidante.

En las paredes se ha seguido el mismo procedimiento que en la planta baja: rejuntar y reponer el revestimiento de mortero de cal, con el mismo criterio de enrasar con el mampuesto.

Se ha procedido a despejar las troneras defensivas, advirtiendo que la estantería existente, que el proyecto preveía colmatar con fábrica de mampostería, era una estructura de la misma época que el muro principal (el aglomerante de cal era idéntico) y tenía unos acabados en el fondo totalmente diferentes del gotelé que recubría los laterales. Ello nos llevó a determinar que era un elemento que pertenecía a la construcción inicial.

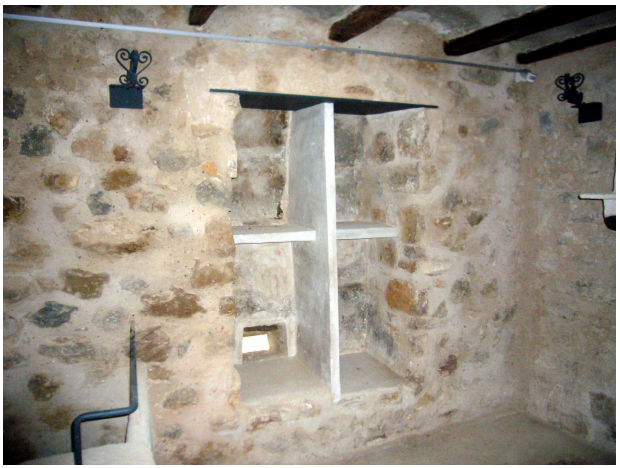

Fig. 8. La estructura de almacenaje interior de la planta primera, antigua estantería (Autor, 2018). 
Tal vez esta estructura formaba parte del mínimo equipamiento que debió disponerse para las necesidades de sus habitantes (cocina y almacenaje) que, obviamente, se ha mantenido en su misma situación, eliminando los elementos clarísimamente contemporáneos.

En este punto existía un peligro estructural cierto por cuanto que las viguetas del techo se habían colocado anormalmente cerca del cierre superior del hueco. Se ha dispuesto un dintel estructural de acero para resolver adecuadamente el apoyo.

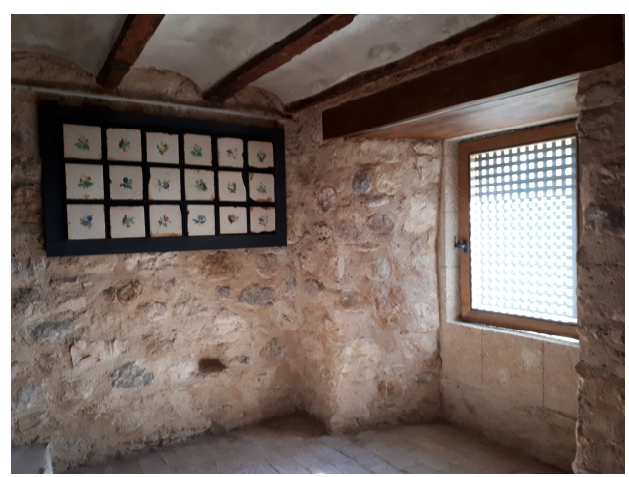

Fig. 9. Posicionamiento definitivo de los azulejos encontrados en el trasdós del balcón suprimido. Son azulejos de finales del siglo XIX pintados a mano sobre un débil tratamiento de serigrafía y habían sido reaprovechados de una ubicación anterior (Autor, 2018).

La chimenea y los techos prácticamente no han precisado intervención alguna, tal solo reparar puntualmente, y entonar cromáticamente el conjunto.

El pavimento de la estancia, que es de ladrillo, se ha saneado y recompuesto con ladrillo manual en los puntos que estaban muy deteriorados o en aquellos puntos donde se incrementaba superficie (espacio resultante del cegado del antiguo balcón).

\section{Entreplanta}

Las catas previas y las operaciones de limpieza iniciales confirmaron que este espacio de la entreplanta se encontraba ya reforzado por su parte superior con una capa de compresión de hormigón, por lo que sobre el suelo del recinto no se intervino y se dio por válido el existente.
El revestimiento de la bóveda estaba resuelto irremediablemente con mortero de cemento, muy involucrado con la propia fábrica. Se eliminó en la medida de lo posible por chorro de arena y se le aplicó una pintura de silicato como acabado. Se realizaron pequeños retoques de afianzamiento estructural y relleno de faltas

\section{Escalera}

Después de las catas realizadas en sus laterales y las tareas de limpieza intensiva que se practicaron, cabe concluir definitivamente que las actuales no son las primeras escaleras del edificio. Seguramente las primeras serían del tipo retráctil (de cuerda y madera) y estarían colocadas en la misma posición que la actuales, pero con un ancho mucho menor.

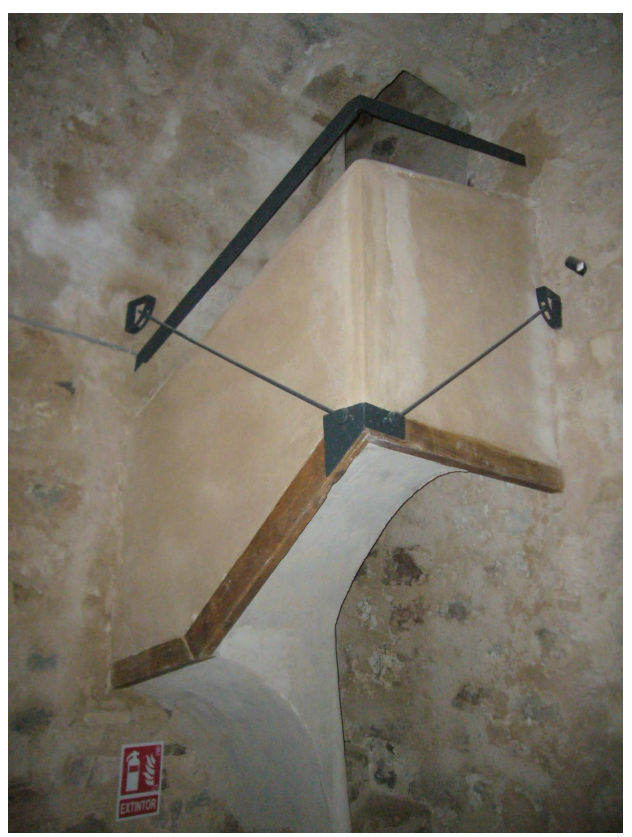

Fig. 10. Acabado final del giro de la escalera de acceso (Autor, 2018).

Es en el momento de gran remodelación interna que tuvo lugar en el siglo XVIII, cuando se decide la introducción de la entreplanta y se configuran las escaleras actuales de trazado helicoidal, para lo que hubo que "empotrar" parte de ellas en el interior de los poderosos muros de cerramiento (tramo de planta baja a primera) o in- 
cluso involucrarlas en el sistema de soporte vertical de los nuevos forjados (tramo de planta primera a cubierta).

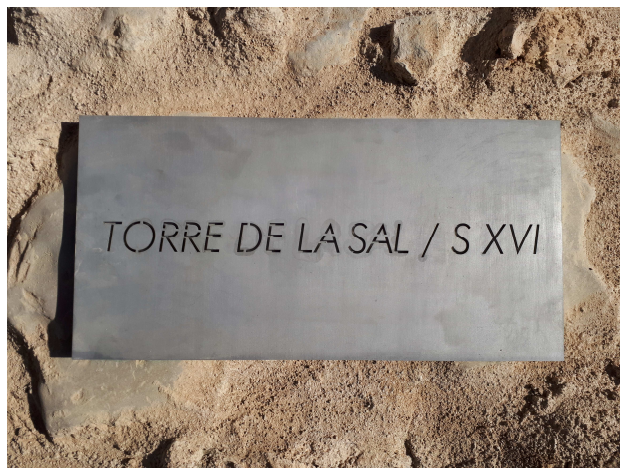

Fig. 11. Señalética de la torre en uno de los mampuestos del suelo, junto a la entrada (Autor, 2018).

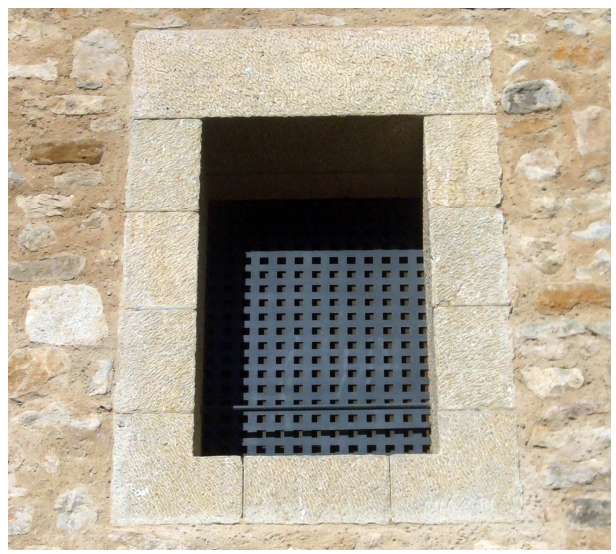

Fig. 12. Detalle del nuevo elemento introducido en el alzado, con proporción áurea en sus trazados interior y exterior del perímetro de la sillería (Autor, 2019).

A nivel de intervención sobre ellas, tan solo mencionar que en las paredes se ha actuado de la misma manera que en el resto de las paredes interiores del edificio (con las que muchas veces está en continuidad): limpieza, eliminación de mortero de cemento y rejuntado con mortero de cal.

Los peldaños de las escaleras están resueltos con un ladrillo macizo de gran calidad. Está agujereado en la tabla con perforaciones cilíndricas de reducido diámetro. Se han aspirado mecánicamente y posteriormente se ha rellenado con arena y/o mortero.
En la planta baja se ha sustituido el tirante vertical existente por el apoyo estructural proyectado, afianzando la componente de tensión en el conjunto. En los puntos donde no se cumplían los mínimos estándares de seguridad se han dispuesto barandillas empotradas resueltas con barras de acero macizo liso de $20 \mathrm{~mm}$.

\section{Instalaciones}

El edificio ha quedado totalmente equipado con una instalación eléctrica, dispuesta empotrada en el momento en que se iban rejuntando las diferentes fábricas. Los mecanismos de iluminación son tiras de LED alojadas en piezas de aluminio lineal sobrepuestas a los muros generando una atmósfera lumínica de baja intensidad pero iluminando especialmente los puntos de paso conflictivo

Se ha realizado también la conexión con las arquetas del sistema de iluminación exterior. Pero en el tiempo que ha trascurrido entre la redacción del proyecto y la ejecución de la obra, la tarea de afección a los intereses públicos no ha cesado. Ya al inicio de los trabajos se pudo comprobar que se había sustraído el cableado de la acometida, dispuesto por debajo del paseo litoral de madera y que alimentaba el sistema de iluminación exterior, por lo que el conjunto ha quedado sin suministro.

Deberá replantearse el Ayuntamiento la conveniencia de instalar un sistema de suministro fotovoltaico que ofrezca la escasa cantidad de energía que se precisa para mantener en funcionamiento las luminarias de LED dispuestas por todo el interior. 

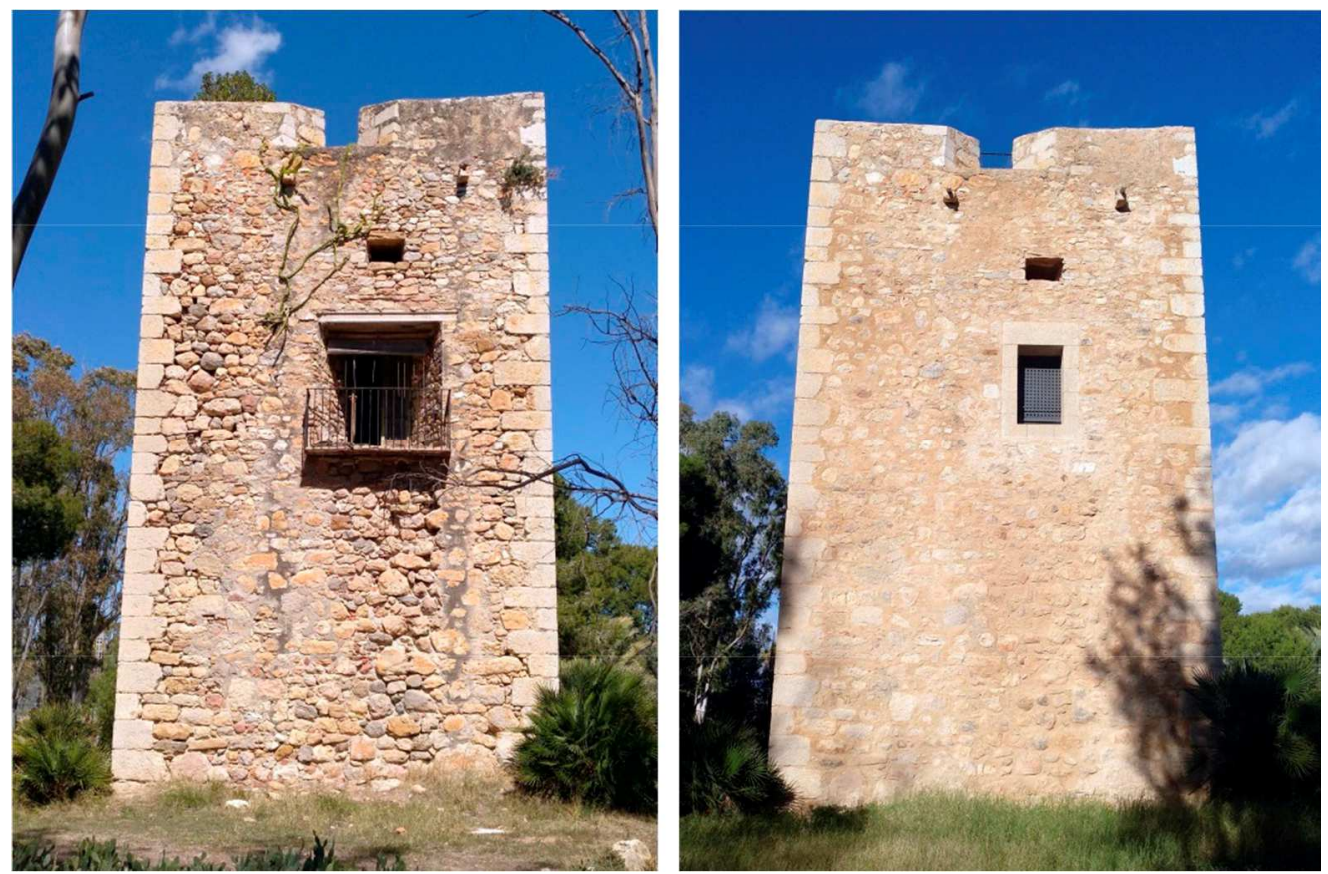

Fig. 13. Vista del alzado sureste (al mar) en su situación inicial y en la final después de la intervención (Autor, 2007, 2019).

\section{Bibliography}

Forcada Marti, V. (1992). Torres y Castillos de la provincia de Castellón, Sociedad Castellonense de cultura Ed., Castellón de la Plana.

Gil Albarracin, A. (2009-2010). "Fortificaciones para la defensa de la costa del Reino de Valencia", Castillos de España, 156-159, pp. 22-48.

Mora-Figueroa, L. de. (1995). Glosario de Arquitectura Defensiva Medieval, Universidad de Cádiz Ed., Cádiz.

Olucha-Montins, F. (1984-85). "Sobre unes torres de defensa litoral”, Revista d'Estudis Castellonencs 2, Castellón, pp. 147-162.

Perales Vilar, E. (1912). Historia de Castellón y Geografía de su provincia, Imprenta de J. Forcada Ed., Castellón de la Plana.

Prior, J. (2018). “«Torre de la Sal» un eslabón en la larga cadena defensiva costera”, in Marotta, A.; Spallone, R., eds., FORTMED 2018. Defensive Architecture of the Mediterranean, Politécnico di Torino Ed., Torino, vol. VIII, pp. 823-830.

Sanchez Adell, J.; Rodriguez Culebras, R.; Olucha Montins, F. (1990). Castellón de la Plana y su Provincia, Diputacion Provincial de Castellón Ed., Castellón.

Sánchez-Gijón, A. (1996). Defensa de costas en el Reino de Valencia, Consell Valencia de Cultura, Generalitat Valenciana Ed., Paiporta.

VV.AA. (2007). La obra castrense en defensa de la costa castellonense, Sociedad Castellonense de Cultura, Obras de investigación histórica, LXXV, Castelló de la Plana. 